\title{
"A new beginning, a raid on the inarticulate " : la critique simonienne en anglais depuis 1989
}

\section{Emelyn Lih}

\section{(2) OpenEdition}

\section{Journals}

Édition électronique

URL : http://journals.openedition.org/ccs/1080

DOI : $10.4000 /$ ccs. 1080

ISSN : 2558-782X

Éditeur :

Presses universitaires de Rennes, Association des lecteurs de Claude Simon

\section{Édition imprimée}

Date de publication : 1 décembre 2017

Pagination : 183-211

ISBN : 978-2-7535-5482-5

ISSN : 1774-9425

\section{Référence électronique}

Emelyn Lih, « "A new beginning, a raid on the inarticulate » : la critique simonienne en anglais depuis 1989 », Cahiers Claude Simon [En ligne], 12 | 2017, mis en ligne le 07 décembre 2018, consulté le 14 février 2020. URL : http://journals.openedition.org/ccs/1080 ; DOI : 10.4000/ccs.1080 


\title{
" A NEW BEGINNING, A RAID ON THE INARTICULATE " : LA CRITIQUE SIMONIENNE EN ANGLAIS DEPUIS 1989
}

\author{
Emelyn LIH \\ New York University
}

Cette étude vient continuer et compléter celle d’Ilias Yocaris, «Un monde qui s'annonce: La réception critique de l'œuvre simonienne dans les pays anglophones ${ }^{1}$ (1959-1989) ", en recensant la critique de langue anglaise consacrée à l'œuvre de Claude Simon et parue lors des vingt-cinq dernières années. Nous prenons notre titre du poème de T.S. Eliot d'où est également tirée l'épigraphe de L'Acacia, Four Quartets ${ }^{2}$. En quoi l'œuvre de Simon et sa critique peuvent-elles être lues comme un "raid dans l'inarticulé " qui permettrait de repêcher de quoi articuler un nouveau langage, un nouveau départ littéraires? La critique de langue anglaise, issue majoritairement du Royaume-Uni et de l'Amérique du Nord, est très abondante dans les années quatre-vingt-dix, mais se tarit quelque peu dans ce début de $\mathrm{xxI}^{\mathrm{e}}$ siècle; nous nous concentrerons ici sur la période comprise entre 1990 et 2010. Comme le fait Ilias Yocaris dans son article, nous ne procéderons pas de manière chronologique mais par ce que nous pourrions appeler des familles

1. Cahiers Claude Simon, $\mathrm{n}^{\circ} 11,2016$, p. 217-259.

2. "And so each venturel Is a new beginning, a raid on the inarticulate/ With shabby equipment always deteriorating/ In the general mess of imprecision of feeling, / Undisciplined squads of emotion " "Et c'est pourquoi chaque tentative/ Est un nouveau commencement, un raid dans l'inarticulél Avec un équipement miteux qui sans cesse se détériore/ Parmi le fouillis général de l’imprécision du sentir,/ Les escouades indisciplinées de l'émotion »), T. S. Eliot, " East Coker », Four Quartets/Quatre Quatuors [1936-1942], dans Poésie (éd. bilingue, trad. P. Leyris), Le Seuil, 1947, p. 182-183. 
critiques, en tentant de décrire l'ensemble du corpus en anglais de cette période à travers l'identification de quelques lignes de force et des différentes orientations d'accent et de méthodologie.

Un point de départ utile est fourni par l'article de Barbara Vinken, paru dans un numéro spécial de la revue Yale French Studies, dont le titre se traduit par "Après l'ère du soupçon: le roman français aujourd'hui ». Rédigé dans l'idiome de la déconstruction, ce court article propose une lecture détaillée de passages des Géorgiques et de Leçon de choses pour faire apparaître leur qualité d' "allégories de la lecture ". Il soutient la thèse formaliste selon laquelle "c'est précisément en mettant l'esthétique au premier plan que [le travail de Simon] est bien plus radical que toute littérature "engagée" 3 " (Vinken 1988: 46 et 48). Cette attention minutieuse au texte lui-même est assez rare dans la critique anglophone en dehors des textes concernés spécifiquement par la traduction vers l'anglais des romans de Simon, en raison sans doute de la difficulté de commenter le dense texte simonien dans une langue autre que le français. Mais il y a plusieurs exceptions à cette règle: nous commencerons donc avec les textes critiques qui se signalent par une attitude de "close reading", mobilisant des méthodes d'analyse tirées de la stylistique et de la linguistique, ou qui revendiquent plus généralement une appartenance à la critique formaliste.

\section{LECTURES FORMALISTES ET LINGUISTIQUES}

Il nous paraît d'abord nécessaire de mentionner la parution en 1993 d'un volume sous la direction de Celia Britton, qui rassemble plusieurs articles importants sur Simon, soit dans leur version originale, comme celui de David Carroll sur Histoire et celui de Lynn Higgins sur La Route des Flandres, soit des traductions vers l'anglais de textes cruciaux dans l'histoire de la critique simonienne (articles de Maurice Merleau-Ponty, Michel Deguy, Jacques Guicharnaud et Jean Ricardou, entre autres). Le recueil est précédé d'une étude introductive de $\mathrm{C}$. Britton qui offre un bref survol des incidences de la vie de Claude Simon sur son œuvre, puis retrace les grands moments de la critique (toutes langues confondues) et déplore le fait que l'attribution du Prix Nobel n'ait pas dissipé l'image de Simon comme étant « un écrivain intransigeant dévoué à l'expérimentation aux dépens de l'accessibilité ${ }^{4}$ »

3. "It is precisely by featuring esthetics that he is far more radical than any "engaged" literature ".

4. Le Nobel, paradoxalement, "perpetuates the general public's view of Simon as an uncompromising writer committed to experimentation at the cost of accessibility". 
(Britton 1993:10). Ce livre participe des tentatives pour faire davantage connaître Simon dans le monde anglophone, et rend service aux critiques en republiant des textes souvent devenus difficiles à trouver, ou qui n'étaient pas disponibles en anglais jusqu'alors.

Parmi les textes consacrés plus directement à un roman en particulier, nous pouvons commencer par un court article d'Yves Clemmen sur L'Acacia. L'auteur propose une lecture du roman de 1989 comme " réponse à un acte de classification », montrant qu'il devient ainsi un " album » où les signes du passé sont sauvegardés au sein de la matérialité rassurante du texte comme document (Clemmen 1992: 126). Il note le silence qui règne dans le roman, plaçant plusieurs scènes sous le signe du visuel, voire du photographique. Ce regard attentif porté aux interférences entre le matériau linguistique et les signes graphiques se retrouve dans l'article de Jean Duffy sur Photographies (l'un des seuls textes critiques qui se concentrent uniquement sur cette œuvre), que nous incluons dans cette section non seulement en raison de son titre ("Claude Simon's Photographies: A Formal and Thematic Analysis »), mais parce que Duffy y insiste sur la " cohérence formelle » de l'album, le beau résultat du mariage d'une grande virtuosité technique et d'une disposition savante des photographies selon des similarités de ligne, de composition et d'éclairage (plus que de sujet ou de motif). Elle glose également la préface à Photographies en situant ses remarques dans le contexte de débats récents sur la photographie, reprenant par exemple l'assertion de Philippe Dubois selon laquelle la photographie est le seul art qui permette de faire un portrait de sa propre ombre, et montrant que c'est précisément ce que fait Simon (Duffy 1994c: 70).

Duffy adopte une démarche semblable dans un article de la même année qui se présente comme une lecture formaliste de L'Invitation. Elle veut battre en brèche et les interprétations réductrices qui ne voient dans le livre de 1987 qu'une simple boutade anti-soviétique, voire anti-communiste, et celles qui, pour trop s'appuyer sur des approches théoriques et linguistiques, peuvent paraître abstruses. Elle donne rapidement les "clefs " de L'Invitation (précisant les identités de Gorbatchev et d'Arthur Miller, entre autres), pour ensuite dénier toute pertinence à cette activité - celle de repérage de personnes connues derrière les épithètes périphrastiques de Simon - et attirer l'attention ailleurs, vers les techniques de défamiliarisation inspirées des formalistes russes à l'œuvre dans le texte: l'omission du nom propre (justement), la promotion du détail, et la construction en boucle. Dans l'analyse de ce 
dernier procédé, elle montre en quoi le récit apparemment linéaire du voyage est constamment traversé par des cycles minuscules, subtile structure en spirale qui rehausse l'aspect baroque du texte et rejette son référent historique dans l'arrière-plan (Duffy 1994b: 453-456). L'absence de noms propres dans L'Invitation attire également l'attention analytique de Lois Oppenheim, qui voit dans l'anonymat une constante à la fois syntaxique et thématique du livre. L'imagination de Simon rappellerait ainsi " le retour phénoménologique aux choses mêmes, par le biais de la suspension même de la référence mimétique " " (Oppenheim 1995: 23). Elle poursuit son analyse en se montrant attentive aux spécificités d'une écriture " aussi répétitive qu'elliptique » (23).

La question du déploiement et de l'ajournement du nom propre intéresse aussi J. Duffy par rapport à L'Acacia. Pourquoi, demande la critique, cette insistance sur de longues périphrases alors même que Simon n'avait jamais prêté beaucoup d'attention à l'anathème que les propagandistes du Nouveau Roman avaient jeté sur le nom propre et sur ses connotations bourgeoises et balzaciennes? À part le fait que la guerre fait vaciller et s'évanouir les velléités d'identité étiquetable chez ceux qui en font l'expérience, Duffy montre que Simon attaque souvent le processus de nomination qui facilite et fausse les récits historiques conventionnels (elle cite le passage de L'Acacia dans lequel les "villages " que visitent les femmes endeuillées sont décrits comme ne correspondant plus aux acceptions du mot village), et que l'intérêt de Simon pour le nom propre porte entièrement sur le plan de la connotation (associative, phonique, graphique), plutôt que sur celui de la référence dénotative simple (Duffy 1995: 37-38).

Un autre article, qui prend comme corpus quelques livres de Simon qui ont un lien avec la guerre d'Espagne, de La Corde raide au Palace en passant par Le Sacre du Printemps, montre la cohérence grandissante entre forme et contenu dans ces textes. Le style du Palace reflèterait ainsi une réalité aussi artisanale que l'est le matériel des milices espagnoles; la rapidité et la perte de la continuité dans le langage font que les cadres de la référence linguistique tanguent et s'entrechoquent comme les taxis de cette ville qui ressemble à Barcelone (Andrews 1989: 148). Mark Andrews emprunte à J. Ricardou le terme de discohérence pour caractériser cet effet de disruption et de reprise, tour à tour, du flux textuel en le reliant à d'autres concepts comme celui, deleuzien, de dysnarrativité, pour rendre compte des aspects cinématographiques du Palace.

5. " $[\mathrm{R}]$ eminiscent of the phenomenological "return to the things themselves", by means of the very suspension of mimetic reference." 
L'un des seuls articles à se concentrer sur un extrait particulier est celui de C. Britton (1998). Elle y commente une longue phrase tirée de la troisième partie des Géorgiques ${ }^{6}$ qui décrit le rituel mensuel de l'ouverture du salon dans l'enfance du cavalier-narrateur tout en fantasmant sur différents ancêtres de "la vieille dame ", et fait minutieusement ressortir à la fois les réminiscences du réalisme et les résistances à celui-ci, à travers le jeu entre les motifs de dissimulation et de dévoilement. Le passage apparaît finalement comme une illustration du talent qu'a l'écriture simonienne d'" activer un niveau secret de sens qui parfois sous-tend, parfois excède, et parfois même va à l'encontre de la diégèse ${ }^{7}$ " (Britton 1998: 199). L'article fournit un bel exemple d'explication de texte sophistiquée. Un autre texte qui lie thème et fonction est la contribution de Joyce Amy Evelyn Loubère à l'ouvrage collectif Claude Simon: A Retrospective, dans laquelle elle suit la trace des manifestations formelles du thème du conflit; cette analyse aboutit à une lecture proprement fonctionnelle d'un élément thématique à travers plusieurs romans de Simon ${ }^{8}$.

Nous terminerons cette section en évoquant deux études inspirées plus directement de la linguistique et de l'analyse du discours. Un article de Dina Sherzer étudie l'emploi de la répétition dans Triptyque ainsi que dans La Maison de rendez-vous d'Alain Robbe-Grillet et L'Observatoire de Cannes de J. Ricardou. Suivre les instances de la répétition lexicale, thématique et syntaxique de la manière la plus concrète se révèle être un moyen tout indiqué pour faire apparaître le va-et-vient entre unité et fragmentation. La répétition agit comme créatrice de redondance, mais par cela même donne une cohésion à ce qui autrement demeurerait épars, créant ainsi (chez Simon en particulier) un effet d'inquiétante étrangeté (Sherzer 1994: 78). L'autre article se concentre sur un phénomène encore plus précis, celui de la parenthèse. On y découvre qu'il y a approximativement 550 paires de parenthèses dans La Route des Flandres, contenant à peu près le quart de tout le texte. Se méfiant de toute généralisation sur la fonction de ces parenthèses, Daniel P. Deneau établit une typologie et mène ensuite plusieurs analyses de détail pour montrer comment la subordination enrichit la prose de Simon, comment les pa-

6. «On retirait les housses [...] de crépusculaires retours de troupeaux » (G, p. 756-758).

7. «Simon's writing activates a covert level of meaning which sometimes underpins, sometimes exceeds, and sometimes even runs counter to the diegesis." "

8. "Que font la guerre et l'érotisme conçu sur le modèle de la guerre? Ils "remuent" [stir up] le texte pour ensuite le briser en morceaux" (Loubère, 2002, p. 97). 
renthèses se substituent aux virgules attendues, et comment elles compliquent le traitement réceptif de la prose, obligeant le lecteur à relire (Deneau 2003: 557). La patiente analyse quantitative et qualitative de ce trait de ponctuation typiquement simonien donne lieu à de riches aperçus interprétatifs.

\section{LECTURES PHÉNOMENOLOGIQUES ET PHILOSOPHIQUES}

Les approches critiques liées à la philosophie et plus spécifiquement à la phénoménologie demeurent présentes dans le paysage de la critique simonienne, même si le moment où cette école d'analyse prévalait n'est plus. En marge de cette attitude se situent des interrogations sur la position de Simon dans les catégories de pensée plus récentes comme celle de la postmodernité. La réflexion de Thomas C. Daddesio s'organise autour de cette question. Il adopte le terme de Linda Hutcheon, " historiographic metafiction", et vise à montrer que les romans de Simon peuvent tous se regrouper sous cette bannière, c'est-à-dire non pas uniquement ceux qui, comme Les Géorgiques, proclament de manière moins ambiguë un retour à l'Histoire; que même Leçon de choses peut être appelé postmoderne. Pour ce faire, il poursuit un parallèle entre différentes problématisations de la notion de vérité depuis Nietzsche; Simon, selon Daddesio, "rejette résolument le dépassement dialectique " des attitudes anglo-saxonne et continentale envers les pouvoirs de représentation du langage 9 (Daddesio 1990 : 80). Nous pourrions aussi mentionner, dans ce prélude aux explorations philosophiques plus approfondies, l'article de Margaret W. Blades, évoqué par Hannes de Vriese dans sa recension de la critique consacrée à L'Acacia ${ }^{10}$, qui veut suivre au plus près le "retour " de Simon depuis les excès d'une position formaliste et "mécanique " vers une exploration des thèmes de la perception et de la réalité (Blades 1991: 36).

Plus poussée est la réflexion de Brian T. Fitch, dans le chapitre qu'il consacre à Simon dans Reflections in the Mind's Eye: Reference and its Problematization in Twentieth-Century French Fiction, qui regroupe également des études sur Beckett, Blanchot et Bataille, entre autres, ainsi qu’une importante introduction sur les théories de la référence dans la fiction. L'introduction établit l'objectif de son livre: faciliter le dialogue entre la poétique et la phénoménologie. Le chapitre sur Simon se concentre sur Histoire,

9. " resolutely rejects [the] dialectical sublation", sublation étant le mot anglais qui traduit le Aufhebung hégélien. 10. H. De Vriese, «L'Acacia, 25 ans de critique », Cahiers Claude Simon n ${ }^{\circ} 11,2016$, p. 27-52. 
et s'ouvre sur une lecture de l'incipit qui associe le célèbre "l'une d'elles » à un " lieu d'indétermination " (dans la terminologie de Roman Ingarden), provoquant un brouillage de l'espace et du temps qui sera radicalisé au moment de la description de la photographie manquée (Fitch 1991: 156). Dans Histoire, le réel s'aplatit dans la mémoire, et le déjà-représenté des cartes postales s'anime. Fitch analyse l'ajournement constant de l'actualisation du référent, report incessant qui achoppe cependant selon lui à la découverte de la réalité matérielle des cartes postales, qui existent réellement dans les archives de l'écrivain - elles agissent donc comme le font les personnages historiques réels dans un roman historique. (Cette conclusion n'est peut-être pas à la hauteur de sa très belle argumentation précédente, qui mêle habilement le Sartre de L'Imaginaire et une panoplie de théories de la réception pour tisser une interprétation magistrale de la fonction des représentations visuelles décrites dans Histoire.)

Il faut enfin évoquer les deux articles importants de J. Duffy qui rouvrent la question trop tôt abandonnée des relations profondes entre l'écriture de Simon et la phénoménologie, en interrogeant les textes de Simon à la lumière de la philosophie de Merleau-Ponty, sans se cantonner aux brefs commentaires que le philosophe a rédigés sur l'écrivain avant sa mort. Par le biais d'un parallèle entre les thèses de Phénoménologie de la perception et celles impliquées par l'écriture simonienne, elle en vient à montrer que la réponse de Simon au béhaviorisme du roman traditionnel équivaut à une "épuration épistémologique (Duffy 1992a: 36) qui permet de voir le monde autrement. Simon et Merleau-Ponty s'intéressent tous deux à des expériences ouvertes, jamais totalisées (Duffy 1992a: 47), et aux circonstances dans lesquelles l'ego peut être amené à se dissoudre - dans des moments de crise, de maladie ou d'excitation érotique - jusqu'à ce que la conscience subjective s'identifie avec son support sensoriel. Dans son second article sur ce sujet, organisé autour de la perception spatiale chez Merleau-Ponty et Simon, Duffy est amenée à interroger les prépositions, qui permettent de mettre en mots les relations spatiales entre les choses, ainsi qu’à énumérer les indices textuels qui permettent d'affirmer que la conscience des personnages chez Simon est toujours incarnée. Ce diptyque sur Simon et Merleau-Ponty accomplit de manière éclatante ce que J. Duffy affirme comme étant son but: "corriger l'impression apocalyptique " proposée par certaines interprétations de Simon, en s'efforçant de « résoudre la contradiction apparente 
entre le chaos ostensible du monde fictionnel de Simon et la cohérence de son approche avec son medium esthétique ${ }^{11}$ " (Duffy 1992b: 72).

\section{Lectures psychanalytiques}

En marge, de nouveau, de cette mince mais importante série d'articles se situent les approches liées à la psychanalyse. Nous ne mentionnerons ici que deux textes, même si la psychanalyse freudienne et surtout lacanienne nourrit aussi la réflexion d'autres critiques, comme Alastair B. Duncan (voir par exemple le chapitre 6 de son Adventures in Words, Duncan 2003) ou Mária Minich Brewer, dont nous parlerons plus loin. Dans la contribution de C. Britton à Claude Simon: A Retrospective, Le Jardin des Plantes est identifié comme le lieu où le traumatisme de l'expérience de la guerre peut finalement être intégré à la normalité et aux catégories de l'exprimable. La distanciation permise par l'enregistrement du journaliste aide à effectuer cette mise à l'écart de la répétition compulsive de la scène de 1940. Peu à peu, au cours du roman de 1997 , le moment traumatique perd son caractère isolé et commence à s'étendre et se diluer parmi d'autres souvenirs (Britton 2002: 74). Aymeric Glacet, dans un court article qui se concentre sur la scène de la photographie manquée d'Histoire, propose que cette section du livre soit interprétée comme "scène primitive ${ }^{12}$ " de l'œuvre (Glacet 2007 : 70), susceptible d'être analysée avec les outils à la fois de la psychanalyse et de l'histoire des techniques photographiques, et notamment des appareils qui prennent la forme d'un fusil - des revolvers photographiques - afin de révéler la présence de la mort dans la photographie du Hollandais.

\section{Lectures historiographiques et mémorielles}

La deuxième catégorie que nous inscrivons sous celle, plus vaste, des approches philosophiques, comprend les textes qui envisagent des œuvres de Simon sous les angles de la mémoire et de l'historiographie. Commençons par un article de William J. Cloonan, repris comme chapitre de son livre The Writing of War: French and German Fiction and World War II. Il y entreprend une analyse de La Route des Flandres comme récit de la tentative

11. "I hope I have gone some way towards correcting the apocalyptic interpretation proposed by some of the early studies of Simon's work and towards resolving the apparent contradiction between the ostensible chaos of Simon's fictional world and the coherence of his approach to his aesthetic medium. "

12. "I have been treating this scene of the studio as the primary scene, indeed, the "primal" scene of the work." 
ratée de Georges d'échapper à l'héritage littéraire, culturel et idéologique du passé. Le personnage de Georges voit clairement l'obsolescence des systèmes de valeurs de son père et du capitaine de Reixach, mais il a bien plus de difficulté à " mettre cette appréhension de la perte en pratique ${ }^{13}$ " (Cloonan 1997: 150). Cette analyse est accompagnée d'une fascinante comparaison entre Georges et Corinne: Georges s'oppose verbalement aux croyances de son père et au souci de sa mère pour son ascendance, mais Corinne agit en rupture réelle avec le monde bourgeois et ses conventions ${ }^{14}$. Cloonan conclut en faisant ressortir le succès du roman La Route des Flandres par contraste avec l'échec du protagoniste. Dans une perspective plus comparatiste, Michael F. O'Riley s'interroge sur les sens historiques de l'étoile (que ce soit la Place de l'Étoile ou le hangar en forme d'étoile entrevu par le protagoniste de L'Acacia lors d'un voyage en train) qui enrichissent ce symbole dans La Place de l'Étoile de Patrick Modiano, L'Étoile errante de J.-M. G. Le Clézio, et L’Acacia. La " place de l'étoile» est ainsi lue comme un chronotope bakhtinien (O'Riley 95) qui renvoie également à la pièce de Robert Desnos qui porte ce même titre. Modiano, Le Clézio et Simon tenteraient d'identifier les coordonnées narratives de ce chronotope.

D. Carroll (2002) s'aventure plus avant dans l'exploration de l'interaction entre histoire et mémoire en prenant comme point de départ le travail de Pierre Nora et son appel à construire une " histoire au second degré ». Antoine Compagnon considère que l'œuvre de Proust constitue en ellemême un lieu de mémoire; pour Carroll, celle de Simon aurait un statut comparable mais distinct. Les romans de Simon mettent constamment en scène la perte de transcendance de la mémoire et les effets de cette perte sur l'expérience vécue de l'histoire (Carroll 2002: 28), une perte non pas appelée à être dépassée de nouveau, mais décrite comme définitive, soulignant l'oublié au cœur même du souvenir (ibid.: 32). Les romans de Simon seraient des lieux de mémoire littéraire où l'histoire est pensée autrement, où la mémoire prend la forme d'une résistance à la mémoire, et la littérature,

13. "Putting this new apprehension of loss into practice, especially when "practice" involves the act of writing, would prove immensely difficult, as Claude Simon well knew. "

14. "[S]he symbolizes a new, inchoate desire for freedom as well as the moral and intellectual emptiness that would characterize the immediate aftermath of World War II" ("Elle symbolise à la fois un nouveau désir mal défini de liberté, et le vide moral et intellectuel qui allait caractériser les lendemains immédiats de la Seconde Guerre mondiale ", Cloonan, 1997, p. 155). 
celle d'une résistance à la littérature ${ }^{15}$ (ibid.: 38). Une approche mémorielle est également décelable dans la conférence de Max Silverman reprise dans le volume collectif Anamnesia: Private and Public Memory in Modern French Culture (2009), intervention dans laquelle la réflexion sur la mémoire est également reliée aux écrits de Walter Benjamin.

\section{LECTURES MARXISTES ET DÉCONSTRUCTIONNISTES}

Nous nous attarderons quelque peu sur cette section, car les perspectives critiques qu'elle décrit n'ont pas à notre connaissance d'équivalent réel dans le champ français des recherches simoniennes, bien que ces perspectives s'inspirent largement de travaux en langue française, de Baudrillard à Derrida. La première critique de notre corpus à revendiquer une perspective marxiste est Doris Kadish. Dans un article de 1993 qui veut faire apparaître le non-dit idéologique des Géorgiques, Kadish propose de lire le roman de 1981 comme une critique filée des trois termes de la devise révolutionnaire "Liberté, Égalité, Fraternité » (1993: 123), et de dévoiler ainsi un parti pris idéologique anti-révolutionnaire et anti-socialiste chez Simon ${ }^{16}$. Ce projet passe en partie par l'expression d'un désaccord à l'égard des analyses de Lucien Dällenbach qui, elles, aboutissent à donner aux Géorgiques le titre de «premier grand roman républicain ${ }^{17}$ ". Cette esthétisation de termes politiques contribue, selon Kadish, à l'escamotage de l'idéologie qui demeure à l'œuvre de manière non reconnue chez Simon. Son article de 1989, paru dans la revue Rethinking Marxism, se concentrait sur Les Corps conducteurs; elle y louait le talent de Simon pour la dramatisation des conflits et des idéologies du présent et du passé récent, même si (d'un point de vue marxiste) une projection positive pour l'avenir fait encore défaut chez l'écrivain (1989: 75-6). Kadish fait référence à plusieurs reprises au critique Fredric Jameson ; nous nous tournerons à présent vers les analyses de Simon par ce dernier.

Le chapitre de Postmodernism, or, the Cultural Logic of Late Capitalism (1991) consacré à Simon est peu cité dans les bibliographies simoniennes. Il s'inscrit dans le vaste projet du livre de Jameson qui reprend son essai

15. " [A] literary site of memory where history is thought "otherwise", a literary site of memory where memory itself takes the form of a resistance to memory and literature takes the form of a resistance to literature."

16. "I shall want to show how Simon's estheticizing ideology can be explained in Les Géorgiques as stemming from an anti-revolutionary, anti-socialist ideology that deviates in significant ways from the values traditionally associated with the Left, with which the New Novel has been commonly thought to be affliated."

17. L. Dällenbach, Claude Simon, Le Seuil, coll. «Les Contemporains », 1988, p. 150. 
de 1984 sur le postmodernisme en l'accompagnant d'une série d'analyses de différents objets culturels, dont, de manière peut-être surprenante, Les Corps conducteurs. Jameson affirme dans la préface qu'il voit dans le Nouveau Roman la dernière innovation importante dans le roman - tout en disant également que le roman comme genre perd de sa pertinence dans l'ère du postmoderne. Le chapitre 5, "Lecture et division du travail ", consacré en grande partie à Simon, s'ouvre sur une question familière de périodisation: les « bizarreries expérimentales » du Nouveau Roman étaient-elles des signes avant-coureurs du postmodernisme, ou une répétition tardive, déjà démodée, d'un modernisme moribond ${ }^{18}$ (Jameson 1991: 131-132)? La réponse à cette question est compliquée par le fait que la relation de Simon aux deux matrices narratives, personnelle et impersonnelle (qui correspondent à deux des phases de son écriture), est celle du pastiche: un pastiche, vrai morceau de bravoure, au point d'en arriver à la reproduction indétectable de l'authenticité stylistique elle-même (ibid.: 133). Les deux phases sont d'abord celle, plus " moderne ", qui pastiche notamment nombre de techniques d'écriture faulknériennes, et ensuite celle d'un collage néo-romanesque bricolé à partir d'une panoplie de genres " naturels " pour créer un genre hybride. La leçon à tirer des romans de Simon des années soixantedix est celle de l'échec du langage, échec à la fois de la nomination et de la deixis et qui reprendrait, dans l'espace de la fiction, l'espace décrit dans le premier chapitre de la Phénoménologie de l'Esprit de Hegel (ibid.: 138). Nous n'avons pas le loisir, dans le cadre de ce survol, de suivre l'analyse détaillée de Jameson; nous nous contenterons de dire qu'elle aboutit à la possibilité que la lecture d'un roman comme Les Corps conducteurs - une lecture nécessairement mot à mot, où production et consommation du sens coïncident - pourrait fournir un analogon du travail non aliéné et de l'expérience utopique d'une société radicalement différente (ibid.: 146).

Il sera également difficile de rendre justice en quelques phrases au travail de M. Minich Brewer dans sa monographie Claude Simon: Narrativities without Narrative. Ce livre veut " réévaluer la force et le potentiel du paradigme narratif pour la compréhension culturelle et critique ${ }^{19}$ " à travers quatre études approfondies d'aspects de l'œuvre de Claude Simon (Minich

18. "Were the experimental peculiarities of the nouveau roman already a harbinger of postmodernism or a belated, already outmoded rehearsal of a dying modernism?"

19. "This study reevaluates the force and potential of the narrative paradigm for cultural and critical understanding by considering the workings of narrativity in [Claude Simon's] writing. " 
Brewer 2005: xi). Son titre défend l'idée qu'une "narrativité sans récit ", conçue comme un démantèlement du récit traditionnel qui réside non pas en son abandon, mais en une exploration approfondie de sa nature, rendrait explicites les résistances que peuvent offrir les textes littéraires aux objectifs hégémoniques des grands récits d'aujourd'hui (ibid.: xvii). Son premier essai retrace le va-et-vient entre des structures narratives empruntées au mythe et à la tragédie, et celles soumises à des temporalités plus aléatoires, dont celle du vent (et du vent qui fait rage dans la ville où se démène Antoine Montès).

Le deuxième chapitre fait valoir le travail de re-figuration de legs narratifs et culturels chez Simon (particulièrement dans Histoire et Les Géorgiques), dessinant la tension entre l'Histoire en tant que répétition et redoublement d'un côté, et différence, division et dissimulation de l'autre (ibid.: 66). Minich Brewer y fait appel à la figure du chiasme telle qu'elle est théorisée par Derrida, et à la structure de renvoi de l'extérieur vers l'intérieur, renvoi évident dans Les Géorgiques dans la collision entre la généralité de la loi et ses effets particuliers sur le frère du général, et sur la succession de ce dernier (ibid.: 70). Les deux chapitres finaux, sur la parodie et sur la «mobilisation" dans tous ses sens, continuent de contrer une lecture anhistorique ou dépolitisée des romans de Simon, et de montrer comment l'esthétique simonienne du détail met à mal les complicités profondes entre écriture et violence et entre la littérature et l'arbitraire du pouvoir (ibid.: 145). Sa contribution au volume collectif d'A.B. Duncan et de J. Duffy (Minich Brewer 2002) va dans le même sens, interrogeant les images de choc et de collision chez Simon afin de caractériser son art poétique comme étant de révolte selon le sens que donne Kristeva à ce concept: Simon résiste - en la représentant - à l'uniformisation de la production de l'image et du consumérisme (ibid.: 56).

Nous terminerons cette section en évoquant deux articles du critique australien Anthony Macris. Le premier reprend la « mise en abyme générative " nommée et théorisée par L. Dällenbach pour en faire un concept plus à même de révéler les "stratégies scripturales » qui détaillent les transformations sociales et industrielles qui ont abouti à la montée des forces du marché et à la pénétration accrue de la marchandisation dans la vie quotidienne (Macris 2003: 51). Tout comme M. Minich Brewer, il emploie le concept de simulacrum de Jean Baudrillard pour décrire les techniques de démystification à l'œuvre dans Les Corps conducteurs, et comme elle également, il loue Simon pour la complexité de son intégration de ces simulacres dans le texte 
(une conséquence de l'intérêt du romancier pour "l'interpénétration des mondes mental et matériel ${ }^{20}$ ", p. 54). À travers une belle analyse d'un passage des Corps conducteurs où le personnage aperçoit depuis son avion toute une foule de forces naturelles et humaines à l'œuvre ${ }^{21}$, Macris en vient à proposer l'idée d'une " poétique matérialiste » chez Simon (" materialist poetics", p. 58). Il est intéressant de voir à quel point Les Corps conducteurs, le « roman américain » de Simon, exerce une attraction particulière sur tous les critiques évoqués dans cette section; mais les analyses de Macris montrent aussi, par exemple, comment le registre de Marie dans L'Herbe fonctionne comme synecdoque du cycle de production et de consommation capitaliste (ibid.: 61-62). L'autre article d'A. Macris décline également les possibilités interprétatives de la mise en abyme (de duplication paradoxale, dans ce cas), à travers une comparaison fructueuse avec des mises en abyme pratiquées par Beckett dans Malone meurt (Macris 2004).

\section{LECTURES INTERTEXTUELLES}

Cette section se concentrera sur des travaux de Mary Orr et de J. Duffy. La première grande référence en la matière est le livre de $\mathrm{M}$. Orr, qui conclut en disant que l'œuvre de Simon peut être vue comme un véritable paradigme de l'intertextualité sous toutes ses formes, à toutes les échelles, y compris à travers l'autocitation (Orr 1993: 203). Sa monographie est structurée de manière originale: elle y entreprend une traversée chronologique, non pas de l'œuvre de Simon, mais des sources de ses emprunts intertextuels, depuis les auteurs de l'Antiquité jusqu'aux grands romanciers modernes du $\mathrm{xx}^{\mathrm{e}}$ siècle et aux allusions intratextuelles que Simon fait à ses propres romans antérieurs. Dans chaque chapitre, elle prend une différente théorisation de l'intertextualité comme guide, en commençant avec une étude classique des sources et des influences et en passant par des conceptions structuralistes, kristeviennes, bakhtiniennes, et ainsi de suite, sans oublier Gérard Genette ni les théories de "l'anxiété de l'influence » de Harold Bloom.

Son livre a le grand mérite d'examiner attentivement des intertextes très peu analysés ailleurs - dont la présence importante de La Dévotion à la Croix

20. "[U]nlike Baudrillard, Simon does not stand wistfully on the shore of idealism, watching a sea of situations drown itself. He is much too interested in how the mental and material worlds interpenetrate to take up such an easy position."

21. «Dans la nuit, d'avion on peut voir de loin en loin [...] des millions de tonnes d'eau basculant avec fracas dans les sauvages cataractes " $(C C$, p. 466-467). 
de Calderón dans Le Vent (Orr 1993: 51-54) - et la complexité de l'insertion des passages de Dix jours qui ébranlèrent le monde de John Reed dans Histoire. En outre, Claude Simon: The Intertextual Dimension mentionne la pièce La Séparation, les fragments de roman publiés dont "Babel " et "Correspondance ", et tout le corpus (déjà très vaste en 1993) des textes et entretiens publiés dans la presse ou dans des revues. L'ampleur de son objet n'empêche pas la critique de faire des analyses détaillées, pour montrer par exemple comment Les Géorgiques "suppriment les connotations idylliques " ("delete the idyllic overtones", p. 27) des Géorgiques de Virgile. En lisant son chapitre sur Proust et Simon, le lecteur se demande comment Orr aurait interprété l'important intertexte proustien du Jardin des Plantes dans son analyse; une réponse partielle est fournie par son article de 2002, qui glose la présence de Proust et de Flaubert dans le roman de 1997 tout en proposant un intertexte jusqu'alors ignoré, à savoir la nouvelle « Le jardin aux sentiers qui bifurquent " de Jorge Luis Borges, pour arriver à une lecture de l'inter- et de l'intra-textualité comme forme d'espionnage (Orr 2002: 122).

Si nous élargissons un tant soit peu la notion d'intertextualité pour y inclure des formes de métissage générique, $M$. Orr demeure une référence obligée. Dès 1988 et 1990, elle a travaillé sur certains des intertextes si nombreux des Géorgiques: le "pont » interdiscursif établi entre la mention " roman » sur la page de couverture et la référence au poème épique imposée par le titre virgilien, pont qui doit passer par le genre dramatique et la structure en cinq «actes » (Orr 1990: 232), et le curieux passage sur Lytton Strachey au milieu du roman (Orr 1988). Mais c'est J. Duffy qui, dans le domaine anglophone, va le plus loin dans l'exploration du terrain des croisements génériques et discursifs chez Simon. Ce travail commence dès l'article sur L'Herbe qui propose de réexaminer ce roman sous d'autres angles que celui de la lecture formaliste de Gérard Roubichou. Sans entrer dans les détails de son argumentation, nous dirons que Duffy présente une variété impressionnante de preuves (tirées à la fois du texte de Simon lui-même, d'œuvres plus générales de critique littéraire comme les travaux de Tony Tanner et Judith Armstrong sur l'adultère dans le roman, et d'autres théories du conte et du merveilleux, de Propp à Ziolkowski) pour montrer que L'Herbe renvoie dos à dos deux ensembles de conventions littéraires: les normes de la littérature du surnaturel - menaces invisibles, boîtes mystérieuses, miroirs enchanteurs - sont savamment mêlées à celle du roman bourgeois de l'adultère et donc de toute la fiction réaliste du XIx ${ }^{\mathrm{e}}$ siècle (Duffy 1994d: 90). 
Après la publication du Jardin des Plantes, J. Duffy s'est de nouveau intéressée aux coexistences de textes et de genres, cette fois-ci au sein du dernier grand roman de Simon. L'un de ces articles prend pour titre la remarque (défensive? irritée? gouailleuse?) tirée des Démons: "Ce n’est pas une allégorie. C'est une feuille, tout simplement" (JP, p. 943). Duffy veut contrer ou du moins nuancer l'idée que Le Jardin des Plantes n'est qu'une autobiographie à peine voilée, en analysant notamment les différents intertextes tirés de récits autobiographiques et biographiques: elle met en lumière la variété d'attitudes envers le récit de vie d'un artiste contenue dans le roman, depuis la réserve de Brodsky jusqu’à la comédie surjouée de Picasso en passant par l'expression prudente de S. (Duffy 1998a: 588-589). Partout, Duffy montre sa familiarité avec le discours de Simon dans ses déclarations journalistiques et ses entretiens, et affiche sa volonté de soutenir les thèses de ce discours par de patientes analyses. Son attention à la structure complexe du Jardin des Plantes reparaît dans un article qui se concentre sur la manière dont les toiles et les titres de Novelli et de Picasso résonnent à travers tout le roman (Duffy 1999).

C'est également Le Jardin des Plantes qui retient l'attention de David Ellison dans sa contribution à Claude Simon: A Retrospective, à travers une analyse des "récapitulations " du roman: il propose d'appeler l'activité de mise en fiction " a departure from the real" (Ellison 2002: 138) en s'inspirant du mot de $S$. à l'intention du journaliste ("ç'a toujours été plus ou moins à partir de choses que j'ai vécues ${ }^{22} »$ ), et trace un chemin depuis le lieu physique du Jardin des Plantes jusqu'à la gravure de Paul et Virginie sur le mur de la scène à Madras, pour ensuite considérer le sens de la juxtaposition des citations de Proust et de Rommel. Notre dernière entrée dans cette section consacrée à l'intertextualité sera le bel article de J. Duffy sur La Bataille de Pharsale, roman auquel elle revient à l'occasion de l'analyse qu'en propose Thierry Marin dans Pour un récit musical ${ }^{23}$ pour prouver que le roman de 1969 s'inspire davantage de la fugue que de la musique sérielle pour sa structure polyphonique complexe. L'analyse fertile de la fugue dans tous les sens du terme sert de prétexte à une analyse intégrale du roman, dont une énumération impressionnante par son exhaustivité des fonctions des nombreux intertextes (Duffy 2005b : 349). Elle décèle jusqu’à la présence « en mineur " des motifs de la fuite éparpillés dans le roman: la fuite

22. Ibid., p. 955.

23. T. Marin, Pour un récit musical, L’Harmattan, coll. « Critiques littéraires », 2002. 
amoureuse, le topos du tempus fugit, la mise en fuite militaire, la citation de La Fugitive... Arrivé à la fin de cette analyse, le lecteur ne peut que s'incliner devant la conclusion que fugue est le véritable mot-carrefour de La Bataille de Pharsale (Duffy 2005b: 354-5).

\section{LECTURES INTERDISCIPLINAIRES}

Les interprétations qui s'aventurent réellement au-delà des frontières des disciplines littéraires et artistiques sont peu nombreuses, mais offrent un éclairage intéressant sur les possibilités d'analyse scientifique des romans de Simon. La tentative la plus aventureuse est celle de Maureen Troiano, dont la monographie New Physics and the Modern French Novel se donne pour objectif de définir des catégories communes de discours entre la science et la littérature modernes, en s'appuyant sur les romans de Simon, Robbe-Grillet et Blanchot. Son chapitre sur Simon se concentre sur Orion aveugle et La Route des Flandres, examinés comme expressions d'une esthétique qui se fait l'écho d'idées et de dynamiques scientifiques (Troiano 1995: 53). Elle établit des liens entre le point de vue narratif problématique dans le roman moderne et le statut problématique de l'observateur dans la physique moderne, et retrace les aspects entropiques de La Route des Flandres. Libérée des contraintes du temps linéaire, la réalité évoquée dans le texte simonien se lit comme disposant de plusieurs vecteurs qui la font se dilater vers une "multi-dimensionnalité unique" (ibid.: 78). La fonction positive de l'entropie est de développer un "message " (au sens que la théorie de l'information donne à ce terme) nouveau et complexe, contenant en lui-même, dans le cas de La Route des Flandres, des rétractions d'éléments du récit qu'il est en train de tisser (ibid.: 109-110). La mort de de Reixach plusieurs fois reprise serait ainsi un élément de redondance communicationnelle. Les analogies de Troiano sont suggestives, mais semblent parfois reposer sur un emploi très métaphorique des concepts scientifiques mobilisés.

De manière beaucoup plus précise, Andrea Goulet s'intéresse à l'optique dans Triptyque ${ }^{24}$ (Goulet 2000). Commençant par le constat de l'importance de la perception visuelle et de son absence - l'aveuglement, le tâtonnement - dans l'écriture de Simon, elle fait un détour par l'histoire de l'optique pour y signaler le rôle crucial de l'œil de lapin dans la correction des thèses de Kepler effectuée par Hermann von Helmholtz. À travers une 
analyse détaillée de plusieurs passages de Triptyque, dont ceux qui décortiquent les mécanismes de la vision et ceux qui décrivent le lapin mort et privé d'yeux, Goulet en vient à identifier la mort de la petite fille comme étant le "point aveugle" ("narrative blind spot») de Triptyque. À cette exploration des possibilités qu'offrent la technique et la science à l'interprétation littéraire s'ajoute l'article de Wolfram Nitsch publié dans Claude Simon: A Retrospective, qui analyse plusieurs intrusions de la technologie dans des romans de Simon: le magnétophone du Jardin des Plantes, le chemin de fer de L'Acacia et le projecteur du cinéma des Géorgiques. Le train en particulier est à la fois un élément central de la machine de guerre européenne, permettant le déplacement souvent forcé des populations et des troupes, et un catalyseur de l'activité créatrice de son passager, le poussant à considérer des noms de lieux surgis des ténèbres sous la seule lumière poétique ("Culmont-Chalindrey ", cité dans Nitsch 2002: 160). L'article d'A. Glacet cité plus haut, avec son passage en revue des techniques photographiques, pourrait également entrer dans cette catégorie.

\section{LECTURES INTERGRAPHIQUES}

La primauté de l'art visuel dans les préoccupations intermédiales de la critique simonienne est attestée de longue date. La critique en anglais ne fait pas exception, et les chercheurs manifestent un intérêt tout aussi vif que celui de leurs homologues francophones pour ce vaste volet de l'œuvre simonienne. Comme l'écrit J. Duffy dans l'introduction à Reading Between the Lines: Claude Simon and the Visual Arts, si le sujet de la relation entre la fiction de Claude Simon et les arts visuels et plastiques est loin d'être nouveau, il est également loin d'être épuisé (Duffy 1998b: 1). Sa monographie veut dépasser la seule référence cubiste pour explorer en détail ce qui a pu agir comme "générateur " de l'écriture de Simon dans le travail de certains artistes, d'Uccello à Andy Warhol. Les contraintes d'espace nous interdisent encore une fois de détailler davantage le contenu de cet ouvrage; nous nous contenterons de dire que la monographie de J. Duffy demeure fidèle à son effort constant de démontrer la grande force et surtout la cohérence de l'œuvre simonienne, à travers ses aspects " autobiographiques, biographiques, thématiques, culturels, réflexifs" (Duffy 1998b: 324). Résumons à présent les articles de Duffy qui se concentrent sur la relation de Simon à l'œuvre et à la philosophie de quelques peintres en particulier. 
Le premier de ces articles à faire partie de notre période d'étude se présente comme une étude comparative de Simon et de Cézanne. J. Duffy y énumère de nombreux points de contact entre les esthétiques de l'écrivain et du peintre: leur volonté de retravailler un nombre limité de motifs, leur abolition des hiérarchies esthétiques, leur mépris de la vraisemblance et du réalisme serviles ("slavish verisimilitude and realistic motivation"), et leur admiration partagée pour Poussin (Duffy 1992c: 47). Elle offre à l'appui de ces propositions des aperçus issus de la correspondance de Cézanne, dont cette belle phrase qui pourrait effectivement avoir été prononcée par Simon: "Le tout est de mettre le plus de rapport possible » (cité dans Duffy 1992c: 41). Dans son article autour de Jean Dubuffet, J. Duffy cherche à aller au-delà du simple constat de l'amitié et de la correspondance entre Simon et l'artiste pour déceler la source chez Dubuffet de certains éléments narratifs de Triptyque, et elle met en valeur leur affection commune pour des procédés de défamiliarisation et leur culture de l'ambiguïté et de la connotation aux dépens de la clarté référentielle (Duffy 1994a: 113). Son article sur Robert Rauschenberg obéit à une méthodologie similaire: au lieu de se contenter de voir en Charlene le générateur d'Orion aveugle et, partant, des Corps conducteurs, elle pousse le parallèle plus avant, jusqu'à affirmer que l'œuvre de Rauschenberg " constitue l'équivalent graphique de l'entreprise de Simon $^{25}$ " (Duffy 1997: 93). Comme Simon, Rauschenberg aime à incorporer de multiples autoportraits à ses œuvres; les deux hommes voient leur art comme une aventure au cours de laquelle l'écrivain ou le peintre répond à des stimuli imprévus, qui peuvent venir infléchir la forme de l'œuvre (ibid.: 96). Enfin, dans sa contribution personnelle à Claude Simon: A Retrospective, Duffy entreprend une analyse détaillée des traces que laisse la toile de Poussin La Peste à Asdod dans Le Jardin des Plantes (Duffy 2002).

Robert Buch explore également la présence des arts visuels dans un livre de Simon en particulier: dans son cas, ce sont les images violentes de La Bataille de Pharsale (Buch 2001). À travers une lecture attentive de certains passages ekphrastiques du roman, Buch réussit à montrer comment la présence d'œuvres d'art sert à remplacer une lecture narrative de l'Histoire par une lecture imagée. Chez Simon, les « expériences historiques dévastatrices [...] sont accompagnées, reflétées, mais aussi fragmentées et rendues

25. Le travail de Rauschenberg «constitutes a pictorial equivalent of Simon's own enterprise ». 
opaques par une confrontation avec des œuvres d'art 26 " (Buch 2001 : 27). Dans le chapitre beaucoup plus étendu qu'il consacre à Simon dans son livre The Pathos of the Real, R. Buch approfondit son analyse, en y intégrant notamment des considérations liées à la réception: "l'expérience de lire Claude Simon prend une allure qui imite l'oscillation entre compréhension et confusion, entre un certain degré de reconnaissance et le sentiment d'être complètement perdu ${ }^{27}$ "(Buch 2010: 65). Il commente le statut fertilement paradoxal de cette écriture à la fois ekphrastique et iconoclaste.

Les travaux d'Ari J. Blatt, enfin, retournent à Triptyque pour essayer d'écarter des lectures anhistoriques et y identifier les traces d'une identification latente avec un "présent de l'histoire de l'art " "art-historical present", Blatt 2006: 69). En mobilisant des théories de J. Baudrillard et de Rosalind Krauss, il lit dans Triptyque le désir de transcender la littérature pour arriver à quelque chose de " plus concret et de plus concrètement visuel ${ }^{28}$ " (ibid.: 75). Le livre de Blatt intitulé Pictures Into Words: Images in Contemporary French Fiction poursuit l'analyse de textes de Simon, de Georges Perec, de Pierre Michon et de Tanguy Viel pour mettre en lumière leur caractère d'iconotexte ou d'image-texte (Blatt 2012).

\section{LECTURES TRADUCTOLOGIQUES}

La traduction vers l'anglais des romans de Simon présente nombre de défis qui n'ont pas manqué d'intéresser les commentateurs anglophones de son œuvre. Marilyn Gaddis Rose a consacré deux articles à sa tentative de discerner la raison pour laquelle l'œuvre de Simon n'a jamais eu de véritable succès auprès du lectorat anglophone; elle a correspondu avec John Fletcher et Richard Howard, traducteurs importants de Simon, et analysé leurs versions des Géorgiques et de La Route des Flandres, entre autres. Mais les faits sont là - les romans de Simon n'ont pas suscité de retentissement autre que " cérébral et tiède" (" a cerebral and tepid response", Gaddis Rose 1989: 175). Elle conclut (dans son second article également; voir Gaddis Rose 1993) que les traductions ne sont pas à blâmer pour ce manque de succès.

26. "Devastating historical experiences [...] are accompanied, reflected but also fragmented and rendered opaque through a confrontation with works of art. "

27. "The experience of reading Claude Simon thus takes on a rhythm that mimics the oscillation between understanding and confusion, between a certain degree of recognition and a feeling of being utterly lost. "

28. "Simon's novel manifests a desire to break free from the material constraints of literature in an attempt to become something other, something more concrete and more concretely visual. " 
La réponse résiderait, selon elle, en la teneur foncièrement négative, voire nihiliste, de l'œuvre du romancier (nihilisme qu'elle ne voit pas chez Gide, Mauriac ou Beckett, pour reprendre ses propres exemples). Mais la question reste ouverte, nous semble-t-il.

J. Fletcher lui-même s'est exprimé sur les fortunes et les faiblesses de sa traduction des Géorgiques: dans un article éloquemment intitulé "A Translator's Second Thoughts ", il revient sur la première page de la première partie des Géorgiques (dont le texte est reproduit, avec sa traduction en regard) pour répondre aux reproches stylistiques qui lui ont été faits en défendant certains de ces choix et en admettant le bien-fondé de quelques remarques critiques qu'il a reçues (Fletcher 1990). De manière moins proprement traductologique, $\mathrm{M}$. Orr s'intéresse à la traduction comme thème et comme processus dans cinq romans de Simon - depuis le latin dans La Bataille de Pharsale jusqu'aux interprètes maladroits de L'Invitation. Dans Les Corps conducteurs et dans la reprise des discours prononcés par des révolutionnaires russes dans Histoire, la répétition et le doublage linguistique interrompent de manière "idéale et ironique " tout discours de propagande ou d'exhortation, ce qui convient à merveille au projet d'écriture de Simon à leur endroit (Orr 1989: 67). Dans ces deux cas, comme lors du pénible congrès des Corps conducteurs, la traduction " mot à mot " agit comme facteur de retardement et d'obscurité, qui annule l'efficacité de la parole en langue étrangère au lieu de la clarifier.

\section{INTRODUCTIONS À L'CEUVRE DE SIMON}

Pour faire face, justement, au manque relatif de familiarité avec l'œuvre de Simon des lecteurs anglophones, il est impératif de disposer d'ouvrages et d'articles qui facilitent l'approche d'un écrivain parfois difficile d'accès. Le premier livre de ce type est Understanding Claude Simon de Ralph Sarkonak (qui, bien sûr, est également l'auteur d'importantes études critiques sur Simon en langue française, et qui, pour cette raison, ne relèvent pas de notre corpus). Il commence son ouvrage en rapportant des anecdotes (très drôles) sur la perplexité des journalistes et de l'intelligentsia nord-américaine au moment de l'attribution du prix Nobel à Claude Simon. Son livre prend pour thèse structurante l'idée que lire Simon nous apprend, chemin faisant, à le lire et à lire tout court. Il traverse en dix chapitres toute l'œuvre depuis Le Vent jusqu'aux Géorgiques. Sarkonak accorde une attention par- 
ticulière aux titres, aux épigraphes ${ }^{29}$, à l'onomastique et généralement à la complexité du tissu textuel simonien. Il n'oublie pas que son lecteur modèle est quelqu'un qui ne connaît pas encore l'univers narratif foisonnant de Simon, et offre ainsi des recommandations utiles sur le meilleur ordre dans lequel lire les romans ou sur des procédés qui reviennent chez Simon (dont la réapparition de personnages de livre en livre, qui sert non pas à créer mais à subvertir un univers fictionnel cohérent, Sarkonak 1990: 155); mais il n’oublie pas de présenter également les principales écoles de pensée dans la critique simonienne et de manifester son accord ou son désaccord avec des interprétations existantes.

Deux chapitres qui prennent place dans des recueils d'essais manifestent aussi leur admiration pour Simon dans l'espoir de convaincre de nouveaux lecteurs de se tourner vers son œuvre. R. Howard, l'un des plus grands traducteurs américains de littérature française, consacre un bel article à Simon au moment du prix Nobel. Après avoir mobilisé son expérience de traducteur pour parler de l' " hypertrophie de l'adjectif » et de la pratique du collage chez Simon, il met l'accent notamment sur la virtuosité de la prose simonienne dans ses relations avec les œuvres d'art de toutes sortes - imaginaires et réelles, statiques et mouvantes (Howard 1995: 160-164). Simon fait aussi partie de ces romanciers de la deuxième moitié du $\mathrm{xx}^{\mathrm{e}}$ siècle dont le critique et traducteur anglais John Taylor brosse un portrait élogieux dans le cadre de sa collection d'essais intitulée Paths to Contemporary French Literature. Il commente rapidement (dans l'idiome du critique littéraire journalistique talentueux) toute l'œuvre jusqu'au Tramway, avec des remarques finales sur le Temps chez Simon: le romancier suggérerait que «l'essence de l'expérience humaine réside dans les tapisseries éphémères que nous construisons à partir du souvenir, de la perception et de l'anticipation ${ }^{30}$ ", trois phénomènes qu'il relie à la conception augustinienne du temps (Taylor 2004: 172). Il faudrait aussi inclure dans cette section l'important livre de synthèse d'A. B. Duncan, mais nous remettons la discussion de Claude Simon: Adventures in Words à notre section finale, car il se situe au moins autant sur

29. Il trace, par exemple, un lien simple mais brillant entre le titre et l'épigraphe du Palace en faisant remarquer qu'ensemble ils font irrésistiblement penser à l'idée d'une "révolution de palais " (Sarkonak 1990: 47).

30. "Simon suggests that the essence of human experience lies in these ephemeral tapestries that we construct out of recollection, perception, anticipation." 
le plan de l'analyse métacritique des œuvres de Simon et des Simoniens que sur celui de la vulgarisation.

\section{REGARDS NOUVEAUX, LECTURES COMPARATIVES}

Cette avant-dernière section rassemble quelques articles importants qui se seraient malaisément intégrés dans les rubriques précédentes, et qui ont en commun le fait de proposer une approche comparative. Dans sa contribution au volume Claude Simon: A Retrospective, A. B. Duncan mène une réflexion autour de l'humour chez Simon, trop peu relevé par la critique, en faisant appel aux travaux de plusieurs théoriciens de la satire et de la réception, de l'hétéroglossie bakhtinienne à la conceptualisation par Wolfgang Iser de la coexistence chez Beckett d'une vision tragique et d'une tonalité comique (Duncan 2002: 111). Il montre, par exemple, que le regard porté par le romancier sur ses personnages est souvent à la fois ironique et admiratif - en d'autres mots, il est à la mesure de leurs efforts à la fois dérisoires et héroïques. Le Jardin des Plantes lui fournit son exemple central, car la présence du comique y est plus sensible, mais il propose également une typologie des instances de satire et de travestissement comiques parmi la profusion de langages dans Le Vent et dans La Route des Flandres (Duncan 2002: 106-110). Quelques années plus tard, J. Duffy prend acte du retour vers le sens et vers certains thèmes - de la famille à la mémoire - dans le discours critique actuel, ce qui autorise l'emploi, dans un contexte d'analyse littéraire, de travaux tirés des disciplines qui parlent du monde référentiel, comme la sociologie ou l'anthropologie de la maladie (Duffy 2005a: 208). L'analyse d'un corpus assez important (comprenant des romans de Jean Rouaud, Laurent Mauvignier et Hélène Renoir aux côtés du Tramway) trace les incidences de l'élément narratif de la maladie sérieuse à travers cet échantillon de la fiction littéraire récente.

Un autre article comparatiste est celui de M. Orr (2005), qui met Le Tramway en parallèle avec Histoire du Tableau de Pierrette Fleutiaux. Elle cite également la chute de bicyclette narrée dans Le Tricheur pour mener son analyse des expériences de confrontation directe avec la mortalité, et celles de la perte de soi, qu'elle prend comme fil pour proposer l'étiquette d'auto-thanatographie (qui permettrait entre autres d'éviter les écueils de l'interprétation autobiographique des œuvres). Enfin, le texte le plus récent de notre corpus est un article consacré majoritairement à Proust sur la re- 
lation entre le mot et le monde qui se dessine dans La Recherche (Hughes 2014). L'auteur fait appel à Simon dans la dernière partie de son travail, saisissant l'occasion du centenaire de Du côté de chez Swann pour examiner l'héritage de Proust chez les grands romanciers qui lui ont succédé. Il met côte à côte une conférence de Simon consacrée à Proust ${ }^{31}$ et les sections du Jardin des Plantes qui reproduisent des extraits de scènes proustiennes avec une typographie novatrice. Il fait apparaître la délicatesse de l'accommodement que ménage Simon entre un référent social pressant et l'expérimentation formelle (Hughes 2014: 212), lui permettant, comme Proust avant lui, de tisser ensemble les fonctions référentielle et expressive de la littérature.

\section{LECTURES MÉTACRITIQUES}

Cette ultime section prend pour objet les contributions critiques qui entrent en dialogue non seulement avec l'œuvre de Simon en elle-même, mais avec le développement varié et souvent conflictuel de la littérature secondaire autour de cette œuvre. Tout au début de cette étude nous citions un article de T. Daddesio; un autre article contemporain du même auteur nomme les trois mouvements de la critique simonienne, selon une catégorisation désormais familière: les critiques d'obédience phénoménologique, formaliste puis post-structuraliste. Daddesio se concentre sur l'instant de transition (ou de début de coexistence) entre les approches rigoureusement formalistes comme celle de Ricardou et celles de critiques se réclamant du post-structuralisme, comme D. Carroll; il veut défendre Simon - même et surtout le Simon des années soixante-dix - contre le reproche de quiétisme (Daddesio 1989: 243). L'introduction à Claude Simon: A Retrospective offre également une traversée de la réception critique depuis les années soixante, au fil de la parution des romans importants de Simon dans la seconde moitié de sa vie (Duffy et Duncan 2002a). Du point de vue de la complétude de notre bibliographie, il convient également de mentionner plusieurs rééditions de textes (qui ne sont pas de nature métacritique) dont la première publication remonte à avant 1989: le livre de C. Britton que nous avons mentionné plus haut (Britton 1993); un article de L. Higgins intégré à son livre New Novel, New Wave, New Politics, qui veut montrer l'" apolitisme politique " des écrivains du Nouveau Roman et des cinéastes de la Nouvelle Vague (Higgins 1996) ; un court texte de Tom Bishop, déjà publié en 1972

31. « Le poisson cathédrale (1980)», QC, p. 9-38. 
mais traduit vers l'anglais pour être inclus dans From the Left Bank (Bishop 1997), son livre de souvenirs et d'impressions critiques.

La dernière entrée dans ce recensement panoramique sera le livre d'A. B. Duncan, publié d'abord en 1994, puis réédité avec certaines modifications, une nouvelle introduction et un chapitre supplémentaire sur Le Jardin des Plantes et Le Tramway en 2003. Il convient bien à la conclusion de notre étude parce que tout en étant un livre d'introduction aux romans de Simon - là où Sarkonak suggérait aux nouveaux adeptes de commencer par L'Herbe, Duncan propose d'entrer dans l'œuvre avec L'Acacia ou Le Tramway - il adopte tour à tour différentes attitudes méthodologiques envers son sujet, suivant le déroulement de l'œuvre en même temps que celui de sa critique, depuis le "réalisme phénoménologique " "phenomenological realism") des années soixante dans le chapitre sur les premiers grands romans (Le Vent, L'Herbe et La Route des Flandres) jusqu'à une lecture des derniers romans de Simon qui fait valoir leur "cohérence sans clôture " (Duncan 2003: 156). Dans ce chapitre final, il montre en quoi Le Jardin des Plantes dessine un juste milieu entre le refus ricardolien de la connexion entre la littérature et la vie, et l'insistance du journaliste sur leur confusion. À travers le volume, des images font ressortir la cohérence complexe de l'œuvre de Simon. Ainsi celle du ballottement: des personnages dans Le Vent; des narrateurs dans les romans qui vont de L'Herbe à Histoire (voire jusqu'aux Corps conducteurs, avec son portrait partiel d'un homme malade); arrivé à Triptyque, c'est le lecteur qui se trouve à la dérive (ibid.: 57). Partout, Duncan affirme la force égale des deux pulsions créatrice et destructrice - d'images, d'histoires, de sens - à l'œuvre chez Simon.

En effectuant cette traversée de plus de vingt-cinq ans de critique, nous espérons avoir présenté de manière claire et complète le travail des auteurs qui ont accepté le défi d'approcher l'imposante œuvre simonienne avec les outils remarquablement variés de l'analyse littéraire. Nous appelons également de nos vœux un renouvellement dans la critique en anglais de cette œuvre, qui mérite toujours d'attirer l'intérêt des chercheurs d'outreManche, d'outre-Atlantique et d'ailleurs. 


\section{BIBLIOGRAPHIE DE LA CRITIQUE EN ANGLAIS DEPUIS 1989}

Mark Andrews (1989), « Narrative Discontinuity and the Warring Image: The Role of the Spanish Civil War in the Novels of Claude Simon ", dans Frieda S. Brown, Malcolm Alan Compitello, Victor M. Howard et Robert A. Martin (dir.), Rewriting the Good Fight: Critical Essays on the Literature of the Spanish Civil War, East Lansing, Mich., Michigan State University Press, 1989, p. 147-160.

Tom Bishop (1997), From the Left Bank: Reflections on the Modern French Theater and Novel, New York, New York University Press, 1997.

Margaret W. Blades (1991), "Claude Simon's Theory of Perception and Reality in L'Acacia ", The Language Quarterly, vol. 29, no 3-4, 1991, p. 32-43.

Ari J. Blatt (2012), Pictures into Words: Images in Contemporary French Fiction, Lincoln, Nebraska, University of Nebraska Press, 2012.

- (2006), "Towards an Iconology of the Text: Art History and the Expanded Field in Claude Simon's Triptyque ", Word \& Image, vol. 22, no 1, 2006, p. 68-76. Mária Minich Brewer (1995), Claude Simon: Narrativities without Narrative, Lincoln, University of Nebraska Press, 1995.

_ (2002), " (In)Commensurabilities: The Childhood of Events and the Shock of Encounter in Claude Simon ", dans Duffy et Duncan (dir.) 2002, p. 39-60.

Celia Britton (1993), Claude Simon, Londres et New York, Longman, coll. « Modern Literatures in Perspective ", 1993.

- (1998), "Display and Disguise: Textual Activity in a Passage from Claude Simon's Les Géorgiques ", dans Philip Ford and Gillian Jondorf (dir.), The Art of Reading: Essays in Memory of Dorothy Gabe Coleman, Cambridge, Cambridge French Colloquia, 1998, p. 191-199.

- (2002), "Instant Replays: The Reintegration of Traumatic Experience in Le Jardin des Plantes", dans Duffy et Duncan (dir.) 2002, p. 61-76.

Robert Buch (2010), The Pathos of the Real: On the Esthetics of Violence in the Twentieth Century, Baltimore, Maryland, Johns Hopkins University Press, 2010.

- (2001), "Violent Images in Claude Simon's La Bataille de Pharsale », Romance Review no 11, 2001, p. 21-32.

David Carroll (2002), "Thinking History Otherwise: Fiction and the Sites of Memory in Claude Simon ", dans Duffy et Duncan (dir.) 2002, p. 22-38.

Yves W.A. Clemmen (1992), "Claude Simon's Acacia, the Text-Album: Photography and Narrative Construction", Romance Notes, vol. 33, n² 2, hiver 1992, p. $125-131$.

William J. Cloonan (1997), « Memory and the Collapse of Culture: Claude Simon's La Route des Flandres ", Symposium: A Quarterly Journal in Modern Literatures, vol. 51, no 3, automne 1997, p. 146-157.

- (1999), The Writing of War: French and German Fiction and World War II, Gainesville, Florida, University Press of Florida, 1999. 
Thomas C. Daddesio (1989), " History and Formalism in the Writing of Claude Simon ", Romance Languages Annual (RLA), no 1, 1989, p. 239-243.

(1990), «Is Claude Simon a Postmodern Writer? ", Romance Languages Annual $(R L A), \mathrm{n}^{\circ} 2,1990, \mathrm{p} .77-81$.

Daniel P. Deneau (2003), "Reading Simon's (Parentheses) The Flanders Road", Neophilologus, vol. 87, $\mathrm{n}^{\circ}$ 4, 2003, p. 553-561.

Jean Duffy (1992a), "Claude Simon, Merleau-Ponty and Perception ", French Studies vol. 46, $\mathrm{n}^{\mathrm{o}}$ 1, janvier 1992, p. 33-52. (1992b), "Claude Simon, Merleau-Ponty \& Spatial Articulation ", Romance Studies, vol. 10, $\mathrm{n}^{\circ}$ 2, juin 1992, p. 59-73. (1992c), "Claude Simon and Paul Cézanne: A Comparative Study ", Degré Second: Studies in French Literature, $\mathrm{n}^{\circ} 13$, décembre 1992, p. 35-50.

_ (1994a), "Claude Simon and Jean Dubuffet: "Voyageurs Égarés" ", French Forum, vol. 19, no 1, janvier 1994, p. 95-116.

(1994b), "Claude Simon's L'Invitation: A Formalist Reading ", The Romanic Review, vol. 85, n³ 3, mai 1994, p. 437-458.

- (1994c), "Claude Simon's Photographies: A Formal and Thematic Analysis ", Nottingham French Studies, vol. 33, no 2, automne 1994, p. 65-77. (1994d), "Conflicts of Genre in Claude Simon's L'Herbe », Romance Studies, vol. $12, \mathrm{n}^{\circ} 2$, automne 1994 , p. 85-100.

(1995), "Claude Simon's L'Acacia: History as Anonymity, the Name as Word ", The Modern Language Review, vol. 90, $\mathrm{n}^{\circ}$ 1, 1995, p. 29-40.

— (1997), "Cultural Autobiography and Bricolage: Claude Simon and Robert Rauschenberg ", Word \& Image, vol. 13, nº 1, janvier-mars 1997, p. 92-101.

_ (1998a), "Ce n'est pas une allégorie. C'est une feuille tout simplement": Text, Intertext and Biography in Claude Simon's Jardin des Plantes ", The Romanic Review, vol. 89, no 4, novembre 1998, p. 583-607.

- (1998b), Reading between the Lines: Claude Simon and the Visual Arts, Liverpool, Liverpool University Press, coll. "Modern French Writers ", 1998.

_ (1999), "Artistic Biographies and Aesthetic Coherence in Claude Simon's Jardin des Plantes ", Forum for Modern Language Studies, vol. 35 n 2, avril 1999, p. 175-192.

_ (2002), "Truth, Verbiage and Écriture in Le Jardin des Plantes", dans Jean Duffy et Alastair Duncan (dir.) 2002, p. 183-204.

- (2005a), "Illness, Ritual and Liminality in the Post-Nouveau Roman ", Romanic Review, vol. 96, no 2, mars 2005, p. 207-231.

_- (2005b), "Fugue and Flight in Claude Simon's Bataille de Pharsale ", The Modern Language Review, vol. 100, $\mathrm{n}^{\mathrm{0}} 2$, avril 2005, p. 340-355.

Jean Duffy et Alastair B. Duncan (dir.) (2002), Claude Simon: A Retrospective, Liverpool, Liverpool University Press, 2002. 
(2002a), «Introduction: The Critical Reception of Claude Simon since the 1960s », dans Jean Duffy et Duncan (dir.) 2002, p. 1-21.

Alastair B. Duncan (2002), "Satire, Burlesque and Comedy in Claude Simon ", dans Duffy et Alastair Duncan (dir.) 2002, p. 100-117.

_ (2003), Claude Simon: Adventures in Words [1994], Manchester et New York, Manchester University Press, 2003.

David Ellison (2002), "À partir du Jardin des Plantes: Claude Simon's Recapitulations ", dans Jean Duffy et Alastair Duncan (dir.) 2002, p. 135-151.

Brian T. Fitch (1991), Reflections in the Mind's Eye: Reference and Its Problematization in Twentieth-Century French Fiction, Toronto, University of Toronto Press, coll. «Theory/Culture ", 1991.

John Fletcher (1990), "A Translator's Second Thoughts: The First Page of The Georgics", Franco-British Studies: Journal of the British Institute in Paris, ${ }^{\circ} 10$, 1990, p. 107-115.

Marilyn Gaddis Rose (1989), "The Role of Translation in the Reception of Claude Simon ", Meta: journal des traducteurs/Meta: Translators' Journal, vol. 34, $\mathrm{n}^{\circ} 2$, 1989, p. 169-178.

(1993), "Textuality, Intertextuality and Reception: Claude Simon in English », dans José Lambert et André Lefevere (dir.), La Traduction dans le développement des littératures - Translation in the Development of Literatures, Actes du Symposium de l'Association Internationale de Littérature Comparée, Berne, Peter Lang et Leuven University Press, 1993, p. 225-232.

Aymeric Glacet (2007), "Claude Simon's Chronophotograph ", Contemporary French and Francophone Studies, vol. 11, $\mathrm{n}^{\mathrm{o}}$ 1, janvier 2007, p. 69-74.

Andrea Goulet (2000), "Blind Spots and Afterimages: The Narrative Optics of Claude Simon's Triptyque ", The Romanic Review, vol. 91, n³ 3, mai 2000, p. 289-311 (traduit et repris dans ce numéro des Cahiers, p. 79-102).

Lynn A. Higgins (1996), New Novel, New Wave, New Politics: Fiction and the Representation of History in Postwar France, Lincoln, University of Nebraska Press, 1996.

Richard Howard (1995), "Divination by Ashes: An Introduction to Claude Simon ", The Georgia Review, vol. 49, no 1, 1995, p. 160-165.

Edward J. Hughes (2014), "'Les lignes vaines et solitaires de mon écriture": "Word" and "World" in Proust's Recherche", The Romanic Review, vol. 105, no 3/4, mai-novembre 2014, p. 201-213.

Fredric Jameson (1991), Postmodernism, or, the Cultural Logic of Late Capitalism, Durham, North Carolina, Duke University Press, 1991.

Doris Y. Kadish (1988), « New Marxist Criticism and the New Novel: The Example of Claude Simon ", Rethinking Marxism, vol. 1, no 3, septembre 1988, p. 64-77. 
(1993), "Claude Simon and the French Revolution », dans Warren Motte et Gerald Prince (dir.), Alteratives, Lexington, Kentucky, French Forum Publishers, 1993, p. 121-132.

Joyce Amy Evelyn Loubère (2002), "The Dynamics of Conflict in the Novels of Claude Simon ", dans Duffy et Duncan (dir.) 2002, p. 77-99.

Anthony Macris (2003), "Claude Simon and the Emergence of the Generative Mise en Abyme ", Journal of the Australasian Universities Language and Literature Association, vol. 99, $\mathrm{n}^{\circ}$ 1, 2003, p. 50-66.

- (2004), « Samuel Beckett, Claude Simon, and the Mise en Abyme of Paradoxical Duplication ", dans Anthony Uhlmann, Sjef Houppermans et Bruno Clément (dir.), After Beckett: d'après Beckett, Amsterdam, Brill Academic Publishers, 2004, p. 117-130.

Wolfram Nitsch (2002), "Supplementary Organs: Media and Machinery in the Late Novels of Claude Simon ", dans Duffy et Duncan (dir.) 2002, p. 152-167.

Michael F. O'Riley (1999), "The Recoding of La Place de L'Etoile in Modiano, Simon, and Le Clézio ", Romance Notes, vol. 40, n 1, 1999, p. 93-102.

Lois Oppenheim (1995), "Narrating Hi(S)Story: A Brief Commentary on Claude Simon's L'Invitation ", New Novel Review: Nueva Novela/Nouveau Roman Review, vol. 2, no 2, 1995, p. 18-29.

Mary Orr (1988), « Lytton Strachey, Literary Embellishment or Functional Intertext in Claude Simon's Les Géorgiques? ", French Studies Bulletin, no 26, 1988, p. 14-17. (1989), "Mot à Mot: Translation as (Inter)Textual Generator in Five Novels by Claude Simon ", New Comparison: A Journal of Comparative and General Literary Studies, $\mathrm{n}^{\circ} 8,1989$, p. 66-74.

_ (1990), «Intertextual Bridging: Across the Genre Divide in Claude Simon's Les Géorgiques", Forum for Modern Language Studies, vol. 26, nº 3, juillet 1990, p. 231-239.

- (1993), Claude Simon: The Intertextual Dimension, Glasgow, University of Glasgow French \& German Publications, 1993.

- (2002), "The Garden of Forking Paths: Intertextuality and Le Jardin des Plantes", dans Duffy et Duncan (dir.) 2002, p. 118-134.

_ (2005), " Figuring the Mortal Coil: Claude Simon's Le Tramway and Pierrette Fleutiaux's Histoire du Tableau ", Forum for Modern Language Studies, vol. 41, no 4, octobre 2005, p. 386-395.

Ralph Sarkonak (1990), Understanding Claude Simon, Columbia, South Carolina, University of South Carolina Press, 1990.

Dina Sherzer (1994), "Effects of Repetition in the French New Novel ", dans Barbara Johnstone, Roy O. Freedle et Annette Kirk (dir.), Repetition in Discourse: Interdisciplinary Perspectives, I \& II, Norwood, NJ, Ablex, 1994, p. 68-85. 
Max Silverman (2009), «Trips, Tropes and Traces: Reflections on Memory in French and Francophone Culture », dans Peter Collier, Anna Magdalena Elsner, Olga Smith et Emma Wilson (dir.), Anamnesia: Private and Public Memory in Modern French Culture, Berne, Peter Lang, 2009, p. 17-28.

John Taylor (2004), Paths to Contemporary French Literature, t. 1, New Brunswick, New Jersey, Transaction Publishers, 2004.

Maureen DiLonardo Troiano (1995), New Physics and the Modern French Novel: An Investigation of Interdisciplinary Discourse, New York, Peter Lang, 1995.

Barbara Vinken (1988), "Claude Simon: The Stained Paper », Yale French Studies, "After the Age of Suspicion: The French Novel Today », 1988, p. 45-49. 